\title{
IRON DEFICIENCY IN CHILDHOOD OBESITY
}

\author{
Orsolya Olah ${ }^{1}$, Cristina Loredana Pantea ${ }^{1}$, Teofana Otilia Bizerea-Moga ${ }^{1,2}$, Raluca Corina \\ Tamasanu1,2, Raluca Asproniu1, Giorgiana Flavia Brad ${ }^{1,2}$, Vatistas Theodoros ${ }^{2}$ Otilia \\ Marginean ${ }^{1,2}$
}

\section{Abstract}

Introduction: Iron deficiency (ID) is one of the most common causes of anemia in children. Although it is frequently encountered in a clinician's daily practice, the multifactorial etiology of ID still represents a challenge in terms of long-term management. In overweight and obese children, adiposity related inflammation causes ID by decreasing transferrin saturation on one hand and by increasing hepcidin production on the other hand, which in turn decreases iron absorption. Dietary uptake of iron and other micronutrients is also impaired in these children. Aim of the study: To investigate the link between overweight, obesity and iron deficiency in pediatric patients. Materials and methods: A retrospective observational study was conducted over a 1-year period (1st April 2018-31th March 2019), in the Endocrinology Department of "Louis Turcanu" Children's Emergency Clinical Hospital Timisoara. After applying the inclusion criteria, a total of 78 overweight and obese children were enrolled in the study. The Ethics Committee of the "Louis Turcanu" Children's Emergency Clinical Hospital approved the study. Results: The CDC BMI- charts matched for age and sex were used in order to define overweight and obesity (overweight 85 th -95 th and obesity $>95$ th percentiles). According to age patients were divided in 3 groups: toddlers $6 \%$ (age $1-5$ years); preadolescents $40 \%$ (age 5-12 years) and adolescents 54\% (age 12-18 years). Overall, the overweight/ obesity ratio in the studied groups was as follows: 20:80 in toddlers, 13:87 in preadolescents and 45,6:54,4 in adolescents. The highest percentage of ID- patients was found in toddlers $(60 \%)$, followed by preadolescents with half as much patients $(32 \%)$, and the lowest percentages was observed in adolescents (14\%). Although there was a correlation between iron levels and BMI throughout our sample, these obese and overweight children did not associate significant anemia. Conclusions: The negative correlation between BMI and iron levels, even in the absence of anemia, should raise awareness with regard to the micronutrient imbalance that exists in these children from a very young age. Children with elevated BMI may need to be screened for iron deficiency. There is an acute need to expand the studies to establish the cause of ID among children and adolescents. Keywords: obesity, body mass index, iron deficiency

\section{Introduction}

Iron deficiency (ID) is one of the most common causes of anemia in children. Although it is frequently encountered in a clinician's daily practice, the multifactorial etiology of ID still represents a challenge regarding long-term management (1).

Childhood obesity continues to spark the interest of researchers worldwide. It is estimated that the global prevalence of obesity in pediatric patients is $16-31 \%$ (2) However, it has been observed that despite the high prevalence in the western countries, there are countries like Poland and Russia where it is not as frequent (3), a possible explanation could be the easier access to fast-food in develop countries in spite of dietary education.

Excess weight during childhood determines the development of several conditions such as elevated blood pressure and dyslipidemia which leads to cardiovascular disease. Also, type 2 diabetes can develop due to insulin resistance. In short, these patients have a significant increased risk to develop metabolic syndrome. Another issue is the weight gain which leads to breathing issues such as sleep apnea, joint and musculoskeletal problems and, also fatty liver disease (4).

In overweight and obese children, adiposity related inflammation ultimately leads to ID by two different/separate mechanisms: the increase production of hepcidin, TNF- $\alpha$, IL-1, IL-2 and adipokines (resistin and leptin) alters iron homeostasis and iron sequestration in the enterocytes, macrophages and hepatocytes determine a decrease in transferrin saturation (5-8).

It seems that there is also a dietary imbalance, rich in carbohydrates and poor in micronutrients in these children (9). Therefore, obese and overweighed patients are iron deficient. ID usually appears before the onset of anemia.

In preadolescents ID is often linked to a combination of an imbalanced diet, poor in micronutrients/iron with sedentary lifestyle. Adolescents, especially girls, due to increased menstrual bleeding are also at higher risk for ID, as it is known that requirements are yet again increased in this age group (1). Adolescents may also have diminished socio-economic circumstances.

${ }^{1} 1$ st Pediatric Clinic, “Louis Turcanu” Children's Clinical and Emergency Hospital, Timisoara, Romania

${ }^{2}$ Department XI of Pediatrics - 1st Pediatric Discipline, "Victor Babes" University of Medicine and Pharmacy Timisoara, Timisoara, Romania

Email: olah.orsi@yahoo.com,cristina.pantea13@gmail.com,bizerea.teofana@umft.ro,tamasanu.raluca@umft.ro, brad.giorgiana@umft.ro,raluca_asproniu@yahoo.com, theovatistas@hotmail.com, marginean.otilia@umft.ro 
ID has durable effects such as: neurodevelopmental and behavior issues, reduced motor development, disruptions of attention and communication (10). The causes leading to ID are different by age group. Among toddlers, lack of iron in the diet is associated with the increased needs in iron during the fast process of growth as well as a dietary imbalance in consumption of cow's milk.

If the iron requirements are not covered, at the initial stage the iron deposits are reduced, ferritin and iron transferrin saturation are decreased. If the iron balance remains negative, after depletion of iron reserves, hemoglobin, hematocrit decrease followed by reductions of VEM and CHEM, thus installing microcytic hypochromic anemia.

\section{Aim of the study}

The aim of the study is to investigate the link between overweight/obesity and iron deficiency in children and to explore age-specific variations.

\section{Material and method}

A retrospective observational study was conducted over a 1-year period (1st April 2018-31th March 2019), on 78 pediatric patients admitted for overweight or obesity in the Endocrinology Department of "Louis Turcanu" Children's Emergency Clinical Hospital Timișoara.

Inclusion criteria: overweight and obesity as defined by CDC, children aged 1-18 years. Exclusion criteria: genetic and/or iatrogenic obesity.

According to the CDC, overweight is defined as a BMI for sex and age between the 85th and 95th and obesity as a BMI equal or greater than the 95th percentile (11). WHO defines ID as serum ferritin levels $<12 \mu \mathrm{g} / \mathrm{L}$ in children under the age of 5 years and $<15 \mu \mathrm{g} / \mathrm{L}$ in those over 5 years of age, and anemia as a condition in which there is a reduction of either red blood cells, hemoglobin or hematocrit under age-appropriate thresholds. Anemia is defined as Hemoglobin level lower than the normal thresholds as shown in Table 1.

An electronic database consisting of anonymized patient files was created using Microsoft Excel and the correlations between the predictable variable (BMI) and the independent one (Iron level) was assessed.

The retrospective study was approved by the Ethics Committee for Research of the "Louis Turcanu" Children's Clinical Emergency Hospital from Timisoara

Results
A total of 78 pediatric patients admitted for overweight or obesity were enrolled and analyzed in the study, after applying the inclusion criteria. According to age patients were divided in 3 groups: toddlers $6 \%$ (age 1-5 years); preadolescents $40 \%$ (age 5-12 years) and adolescents 54\% (age 12-18 years). The CDC BMI- charts matched for age and sex (12) were used in order to define overweight and obesity (overweight 85th - 95th and obesity $>$ 95th percentiles).

In each group, the incidence of overweight and obese patients according to BMI-CDC charts was analyzed. Thus, obesity in the group of toddlers was $80 \%$, in the preadolescents $87 \%$ and among the adolescents group 54,4\%. Whereas, the complementary percentages of overweight children were as follows: $20 \%$ in the group of toddlers, $13 \%$ in the preadolescents group and among the adolescents 45,6 $\%$.

It was surprising that although obesity predominated in the group of adolescents, an almost equal number of overweight children was found. The highest prevalence of obese patients was observed among children aged 5 to 12 years.

Figure 1 shows the prevalence of ID in the studied groups. The highest prevalence of ID was observed among children 1 to 5 years, with a percentage of $60 \%$. As age increases, the prevalence of ID decreases by approximately a half, $32 \%$ in the preadolescents and $14 \%$ in the adolescent patients, respectively. Therefore, it seems that young age increases the risk for ID.

ID was evaluated through serum iron and ferritin and the relation between adiposity and iron levels was investigated, as depicted in Figure 2.

A negative correlation was observed in all age groups as supported by the slightly decreasing slope of the lines and the negative coefficient representing the slope. However, R2 levels supports a weak negative correlation between studied parameters: in toddlers $\mathrm{R} 2=0,0101$, in preadolescents $\mathrm{R} 2=0,022$ and adolescents $\mathrm{R} 2=0,0189$, as described in Figure 2.

It was also important to evaluate the correlation of hemoglobin $(\mathrm{Hb})$ levels with overweight /obesity because a decrease in $\mathrm{Hb}$ levels defines anemia, and ID is a precursor state. We calculated the prevalence of low $\mathrm{Hb}$ levels in our predetermined age groups. Figure 3 shows $\mathrm{Hb}$ levels among the studied patients according to age groups. Out of the total children up to the age of 5 whose $\mathrm{Hb}$ levels were obtained, considering a threshold of $11 \mathrm{~g} / \mathrm{dL}$, none of them had anemia.

Table 1. Hemoglobin and Hematocrit threshold levels for anemia in children adapted from WHO [7]

\begin{tabular}{|c|c|c|}
\hline Age group & Hemoglobin(g/dL) & Hematocrit $(\%)$ \\
\hline Toddlers (1-5 years) & 11 & 33 \\
\hline Preadolescents (5-12 years) & 11,5 & 36 \\
\hline Adolescents (>12years) & 12 & 34 \\
\hline
\end{tabular}




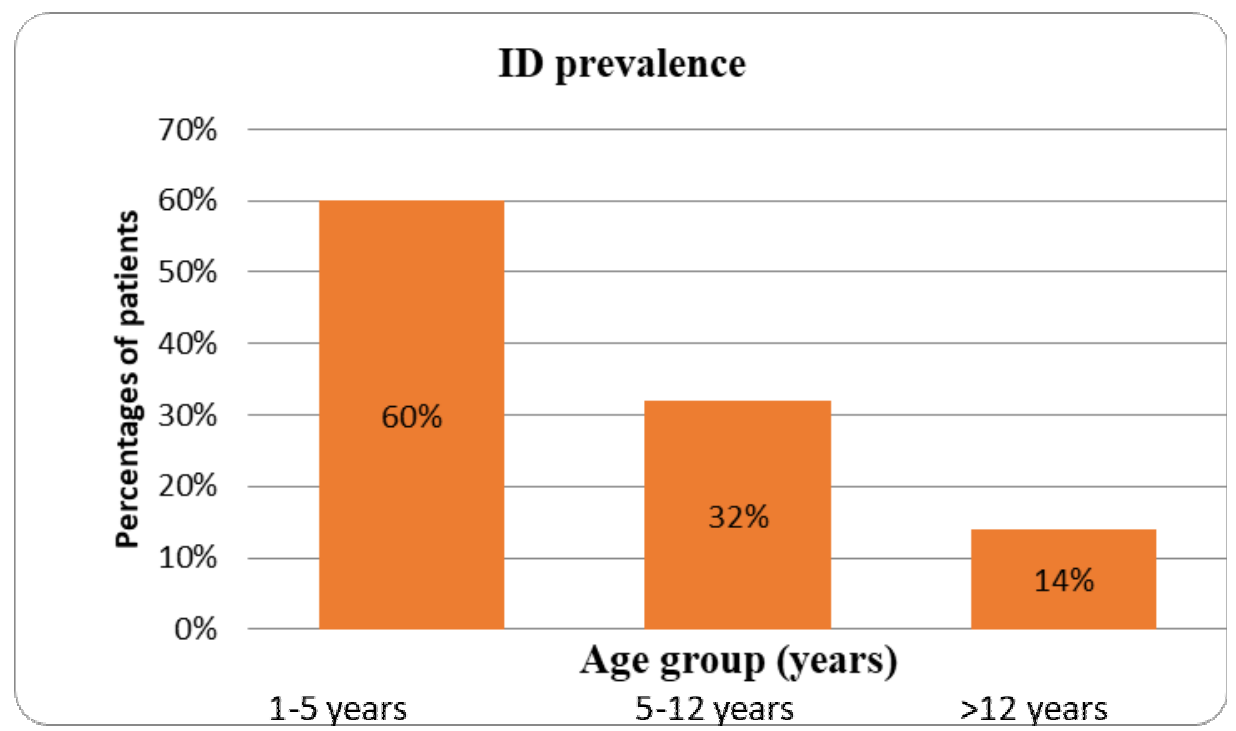

Fig.1. Comparison of iron deficiency between three groups
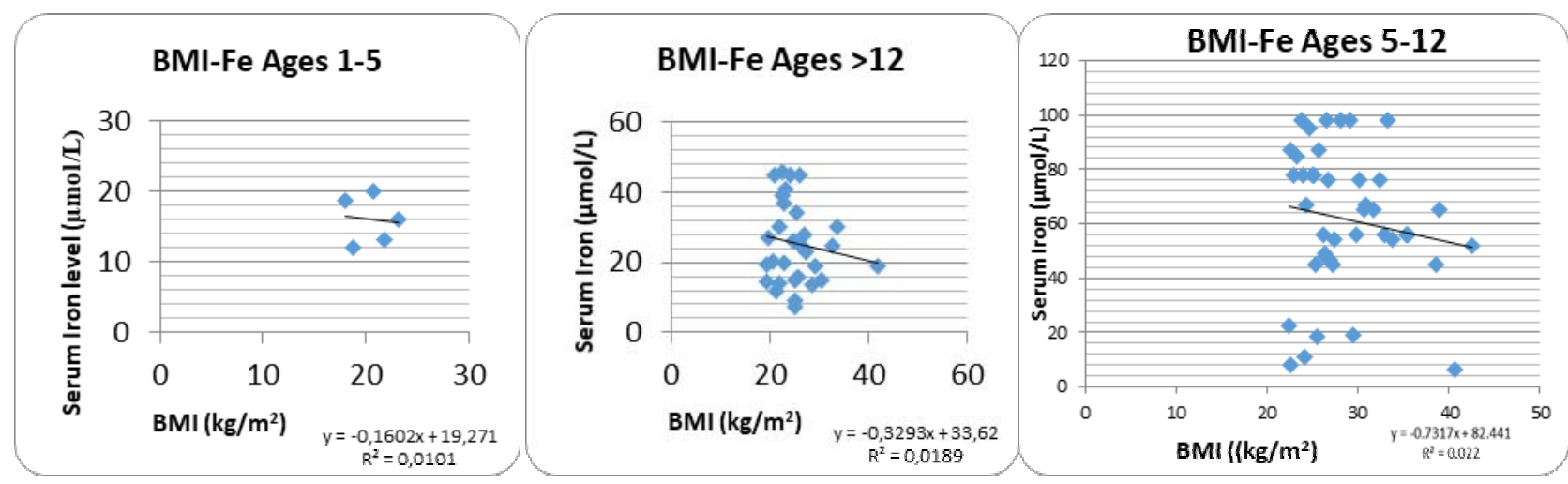

Fig. 2. Correlation of iron levels with BMI in age groups.
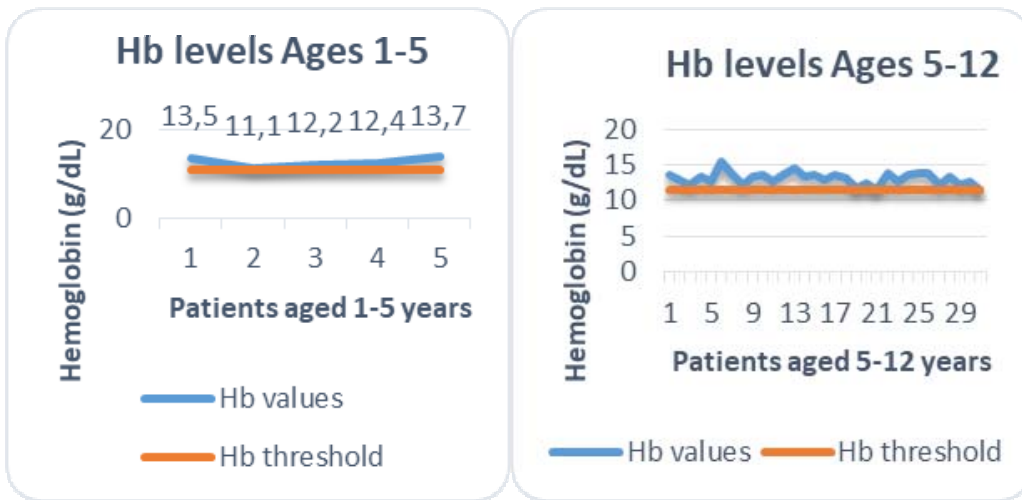

Hb levels Ages $>12$

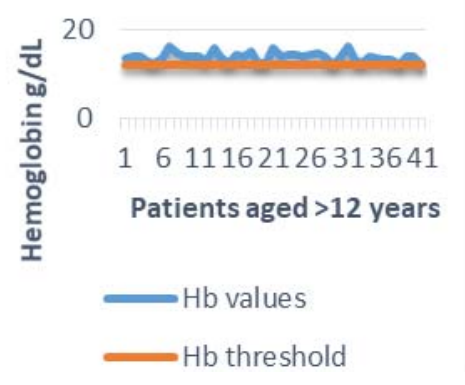

Fig. 3. Hb levels in age groups 
In the preadolescents group, one in 31 children had a decreased level of $\mathrm{Hb}$, while among the adolescents two had low $\mathrm{Hb}$ levels, as depicted in Figure 3.

$3.22 \%$ of obese and overweight children aged 5-12 years had low $\mathrm{Hb}$ level, although there were another 5 patients with $\mathrm{Hb}$ within $0.5 \mathrm{~g} / \mathrm{dL}$ from the threshold. Among adolescents $5 \%$ had $\mathrm{Hb}$ level below the threshold, and 6 adolescents were with $\mathrm{Hb}$ within $0.5 \mathrm{~g} / \mathrm{dl}$ from the threshold. There were no significant differences in $\mathrm{Hb}$ of obese and overweight children between adolescents and adolescent groups. Several of these children may be at risk of developing anemia.

Although iron deficiency was most common in children aged 1-5 years, none had low hemoglobin levels. These results demonstrate that the combination of low serum iron and normal hemoglobin is common among obese and overweight children. In the same time there is a significant number of children throughout our sample with marginal hemoglobin values. The correlation coefficient does not support a connection between BMI and hemoglobin. Thus, it can be stated that negative correlation between BMI and ID does not necessarily imply the same relation between adiposity and anemia.

\section{Discussion}

World Health Organization defines obesity and overweight as a disequilibrium between the calory intake and the consumption (13). In this study it was observed that in the group of children aged 5 to 12 years the proportion of obese patient related to overweight ones was the highest compared to the other studied groups ( $87 \%$ compared to $80 \%$ in toddlers and $54,4 \%$ and adolescents respectively). Obesity and iron deficiency remain two of the most common nutritional disturbances in childhood. Iron deficiency is the principal factor in the development of microcytic anemia (14).

Studies have reported that excess weight has an adverse effect on iron metabolism. In obesity there is a chronic subclinical inflammation associated with reduced iron availability due increased levels of hepcidin and low iron absorption (15-17). There is data that suggests that adipocytes are also capable of producing hepcidin (18). Baumgartner, J, et al. demonstrated that children who are overweight or obese have a two-fold risk of remaining ID after iron supplementation $(19,20)$

An important number of studies have considered that ID in obese pediatric patients is more common than in healthy ones. The data collected by American National Health and Nutrition Examination Survey III, from the pediatric population demonstrated the widespread presence of ID. There are studies that indicate that overweight children from America were twice more likely to be iron deficient than normal-weight children. Thu emphasizing the negative correlation between iron levels and BMI. ID simultaneous rose with BMI $(5,6,9,21)$

In this study obesity was associated with higher ID risk. A negative correlation between BMI and serum iron was observed (toddlers $\mathrm{R} 2=0,0101$, in preadolescents $\mathrm{R} 2=0,022$ and adolescents $\mathrm{R} 2=0,0189$ ).

ID was present in all of the studied groups, but children ages 1 to 5 were the most affected. $60 \%$ of obese and overweight toddlers had ID and the presence was significantly decreased in the other 2 groups, $32 \%$ of the preadolescents and $14 \%$ of the adolescents respectively had ID, whereas the prevalence of decreased by nearly a half in preadolescents and again in adolescents (32\% and 14\% respectively).

Similar results were found in a cross-sectional Canadian study on 1607 children, aged 1-3 years, which concluded that the $\mathrm{z}$-score of BMI was rose inversely proportional to ferritin levels and it was associated with a higher risk of iron deficiency (22). Another study, from China that included over 5000 patients 7-11 years old observed that obesity increased the risk of ID, even though it did not apparently amplify the risk of anemia (23).

A chronic inflammatory caused by obesity leads to hepcidin overproduction which could be the link between excessive adiposity and low iron levels, by decreasing ferroportine- 1 from enterocytes $(24,25)$.It also determines a decrease of transferrin saturation leading to ID.

A study on a large cohort of Greek children showed that obesity increased the risk of ID [20]. However, in children from western societies in spite of an adequate nutritional reserve, the prevalence of ID is significantly increased. This might be due to poor nutritional habits and education, as well as easy access to fast-food (5). Obese children may be at increased risk of micronutrient deficiency (26).

\section{Conclusions}

The negative correlation between BMI and iron levels, even in the absence of anemia, should raise awareness with regard to the micronutrient imbalance that exists in overweight and obese children from a very young age. Children with high BMI may need to be screened for iron deficiency especially in the toddler group.

As iron deficiency has a lot of negative effects on the course of obesity- related conditions, screening of iron deficiency is justified and treatment is warranted for the long- term management. There is an acute need to expand the studies to establish the cause of ID among overweight and obese children and include additional markers such as hepcidin.

Early intervention in case of obesity and routine monitoring of a healthy growth and development are important in order to prevent iron deficiency. In this respect knowing the prevalence of ID among overweight and obese children as a predisposing state for anemia, is important order to counteract its long- term consequences on growth and neurodevelopment $(14,27,28)$. 


\section{References}

1. Powers JM, Buchanan GR. Disorders of Iron Metabolism: New Diagnostic and Treatment Approaches to Iron Deficiency. Hematol Oncol Clin North Am. 2019 Jun;33(3):393-408. doi: 10.1016/j.hoc.2019.01.006. Epub 2019 Mar 29. PMID: 31030809).

2. Ghadimi R, Esmaili H,Kheirkhah D,Tamaddoni A. Is childhood obesity associated with iron deficiency anemia? Caspian Journal Of Pediatrics. 2015;1(2):-.

3. Wang Y, Lobstein T. Worldwide trends in childhood overweight and obesity. Int J Pediatr Obes IJPO Off J Int Assoc Study Obes. 2006;1(1):11-25.

4. Ortíz Pérez M, Vázquez López MA, Ibáñez Alcalde M, Galera Martínez R, Martín González M, Lendínez Molinos F, et al. Relationship between Obesity and Iron Deficiency in Healthy Adolescents. Child Obes. 2020;16(6):440-7.

5. Schmidt PJ. Regulation of Iron Metabolism by Hepcidin under Conditions of Inflammation. J Biol Chem. 2015 Jul 31;290(31):18975-83

6. Sonnweber T., Ress C., Nairz M., Theurl I., Schroll A., Murphy A.T., Wroblewski V., Witcher D.R., Moser P., Ebenbichler C.F., et al. High-fat diet causes iron deficiency via hepcidin-independent reduction

7. Blüher M. Adipose tissue inflammation: a cause or consequence of obesity-related insulin resistance? Clin Sci (Lond). 2016;130(18):1603-14.

8. Aigner E, Feldman A, Datz C. Obesity as an emerging risk factor for iron deficiency. Nutrients. 2014;6(9):3587-600.

9. Nead KG, Halterman JS, Kaczorowski JM, Auinger P, Weitzman M 2004 Overweight children and adolescents: a risk group for iron deficiency. Pediatrics 114:104-108.

10. Mattiello V, Schmugge M, Hengartner H, von der Weid N, Renella R; SPOG Pediatric Hematology Working Group. Diagnosis and management of iron deficiency in children with or without anemia: consensus recommendations of the SPOG Pediatric Hematology Working Group. Eur J Pediatr. 2020 Apr;179(4):527545.

11. Defining Childhood Weight Status- Available online: https://www.cdc.gov/obesity/childhood/defining.html

12. Moschonis, G., Chrousos, G. P., Lionis, C., Mougios, V., \& Manios, Y. (2012). Association of total body and visceral fat mass with iron deficiency in preadolescents: The Healthy Growth Study. British Journal of Nutrition, 108(4), 710-719.
13. Obesity and overweight - Available online: https://www.who.int/news-room/factsheets/detail/obesity-and-overweight

14. Hutchinson C. A review of iron studies in overweight and obese children and adolescents: a double burden in the young? Eur J Nutr. 2016;55(7):2179-97.

15. Richardson MW, Ang L, Visintainer PF, Wittcopp CA. The abnormal measures of iron homeostasis in pediatric obesity are associated with the inflammation of obesity. Int J Pediatr Endocrinol. 2009;2009:713269.

16. Tussing-Humphreys LM, Liang H, Nemeth E, Freels S, Braunschweig CA. Excess adiposity, inflammation, and iron-deficiency in female adolescents. J Am Diet Assoc. 2009;109(2):297-302.

17. Aeberli I, Hurrell RF, Zimmermann MB. Overweight children have higher circulating hepcidin concentrations and lower iron status but have dietary iron intakes and bioavailability comparable with normal weight children. Int J Obes (Lond). 2009;33(10):1111-7.

18. Coimbra S, Catarino C, Santos-Silva A. The role of adipocytes in the modulation of iron metabolism in obesity. Obes Rev. 2013 Oct;14(10):771-9.

19. Baumgartner J, Smuts CM, Aeberli I, Malan L, Tjalsma H, Zimmermann MB. Overweight impairs efficacy of iron supplementation in iron-deficient South African children: a randomized controlled intervention. Int $\mathrm{J}$ Obes (Lond). 2013;37(1):24-30.

20. Zimmermann MB, Zeder C, Muthayya S, Winichagoon $\mathrm{P}$, Chaouki N, Aeberli I, et al. Adiposity in women and children from transition countries predicts decreased iron absorption, iron deficiency and a reduced response to iron fortification. Int $\mathrm{J}$ Obes (Lond). 2008;32(7):1098-104.

21. Gillum RF 2001 Association of serum ferritin and indices of body fat distribution and obesity in Mexican American men: the Third National Health and Nutrition Examination Survey. Int J Obes 25:639-645

22. Sypes EE, Parkin PC, Birken CS, Carsley S, MacArthur C, Maguire J L, Dai DWH. (2019). Higher Body Mass Index Is Associated with Iron Deficiency in Children 1 to 3 Years of Age. J Pediatr. 2019 Apr;207:198-204

23. Zheng, H., Long, W., Tan, W., Yang, C., Cao, M., \& Zhu, Y. (2020). Anaemia, iron deficiency, irondeficiency anaemia and their associations with obesity among schoolchildren in Guangzhou, China. Public Health Nutrition, 23(10), 1693-1702.

24. Abboud S., Haile D.J. A novel mammalian ironregulated protein involved in intracellular iron metabolism. J. Biol. Chem. 2000;275:19906-19912. 
25. del Giudice EM, Santoro N, Amato A, Brienza C, Calabrò $\mathrm{P}$, Wiegerinck ET, et al. Hepcidin in obese children as a potential mediator of the association between obesity and iron deficiency. J Clin Endocrinol Metab. 2009;94(12):5102-7.

26. Ghaemi N, Jafarzadeh M, Bagheri S. Relationship between Obesity and Iron, Calcium and Phosphorus levels in Obese Children 2 - 16 Years Old. medical journal of mashhad university of medical sciences. 2012;55(3):134-8.
27. Tussing-Humphreys LM, Liang H, Nemeth E, Freels S, Braunschweig CA. Excess Adiposity, Inflammation, and Iron-Deficiency in Female Adolescents. Journal of the American Dietetic Association. 2009;109(2):297302.

28. Yanoff LB, Menzie CM, Denkinger B, Sebring NG, McHugh T, Remaley AT, et al. Inflammation and iron deficiency in the hypoferremia of obesity. Int $\mathrm{J}$ Obes (Lond). 2007;31(9):1412-9.

Correspondence to:

Cristina Loredana Pantea

Vadul Crisului 1, 300613

Timisoara, Romania

E-mail: cristina.pantea13@gmail.com

Tel: +40 721246369 